INVESTIGACIÓN

Recibido: 04/10/2017 --- Aceptado: 30/01/2018 --- Publicado: 15/09/2018

\title{
LA INVESTIGACIÓN DE MERCADOS EN REDES SOCIALES: CONOCE A TU CONSUMIDOR POR LO QUE PUBLICA EN FACEBOOK Y TWITTER. ANÁLISIS DE CASO DE ADT SECURITY SERVICES EN CHILE
}

\section{Market research in social networks: know your consumer so publish on Facebook and Twitter. Case analysis of ADT Security Services in Chile}

Beatriz Feijoo Fernández': Universidad de Los Andes. Chile. bfeijoo@uandes.cl

Juan José Guerrero: Universidad de Los Andes. Chile.

jiguerrero@uandes.cl

\section{RESUMEN}

Facebook y Twitter se han convertido en dos ventanas a través de las cuales la sociedad expresa y expone sus opiniones y temores: recientes estudios ponen de relevancia que alguien ajeno puede conocer con más detalle que sus propios familiares a una persona por lo que publica en sus perfiles virtuales (Youyou, Kosinski \& Stillwel, 2015). Situados en este contexto del Big Data, este artículo busca reflexionar sobre la utilidad de las redes sociales como fuente de investigación para conocer, definir y caracterizar al público objetivo, lo que servirá de orientación para el diseño de la estrategia creativa. Se parte del estudio de una campaña de ADT Security Services Chile (TYCO) para la que se empleó como metodología una variante de la etnografía, la netnografía, adaptada al análisis de las comunidades en línea, método más rápido, más sencillo y menos costoso que la etnografía tradicional y más naturalista que los grupos de discusión o entrevistas. Se obtuvo información pública no inductiva sobre los patrones de consumo del target, comprobando así la efectividad del análisis de contenido en redes sociales al conseguir extraer un concepto de seguridad con el que se identificaba el consumidor y sobre el que finalmente se sustentó toda la estrategia creativa.

\section{PALABRAS CLAVE}

Investigación social; Redes Sociales; Facebook; Twitter; Estrategia creativa; Big Data; Netnografía

\section{ABSTRACT}

Facebook and Twitter have become two windows through which society expresses and presents its opinions and fears: recent studies highlight the fact that someone

${ }^{1}$ Beatriz Feijoo: Docente e investigadora en la Facultad de Comunicación de la Universidad de los Andes (Chile). bfeijoo@uandes.cl 
else can know a person in more detail than their own relatives by what they publish in their Virtual profiles (Youyou, Kosinski \& Stillwel, 2015). Focusing on this context of Big Data, this article seeks to reflect on the usefulness of social networks as a source of research to know, define and characterize the target audience, which will serve as guidance for the design of the creative strategy. We are based on the study of a campaign of ADT Security Services Chile (TYCO) for which a variant of ethnography, netnografed, was used as methodology, adapted to the analysis of online communities, a simpler faster, and less expensive method than traditional ethnography and more naturalistic than discussion groups or interviews. Noninductive public information was obtained about the consumption patterns of the target, thus verifying the effectiveness of content analysis in social networks by extracting a security concept with which the consumer was identified and on which the entire creative strategy was ultimately based.

\section{KEY WORDS}

Social research; Social Media; Facebook; Twitter; Creative Strategy; Big Data; Netnography

\section{A INVESTIGAÇÃO DE MERCADOS EM REDES SOCIAIS: CONHECE A TEU CONSUMIDOR PELO QUE PÚBLICA NO FACEBOOK E TWITTER. ANALISES DO CASO DE ADT SECURITY SERVICES NO CHILE}

\section{RESUME}

Facebook e Twitter se converteram em duas vitrines através das quais a sociedade expressa e expõe suas opiniões e temores: recentes estudos põem em relevância que alguém alheio pode conhecer com mais detalhes que seus próprios familiares a uma pessoa pelo que publica em seus perfis virtuais (YouYou, Kosinski\& Stillwel, 2015). Situados neste contexto do Big Data, este artigo busca reflexionar sobre a utilidade das redes sociais como fonte de investigação para conhecer, definir e caracterizar ao público objetivo, o que servira de orientação para o desenho da estratégia criativa. Parte-se do estudo de uma campanha de ADT Security Services Chile (TYCO) para qual se uso como metodologia uma variante da etnografia, a netnografia, adaptada ao analises das comunidades em linha, método mais rápido, mais simples e menos custoso que a etnografia tradicional e mais natural que os grupos de discussão ou entrevistas. Se obteve informação pública não indutiva sobre os padrões de consumo do Target, comprovando assim a efetividade das análises do conteúdo nas redes sociais ao conseguir extrair um conceito de segurança com o qual se identificava o consumidor e sobre o que finalmente se sustentou toda a estratégia criativa.

\section{PALAVRAS CHAVE}

Investigação social; Redes sociais; Facebook; Twitter; Estratégia criativa; Big Data; Netnografía 


\section{Cómo citar el artículo}

Feijoo Fernández, B.; Guerrero, J. J. (2018). La investigación de mercados en redes sociales: conoce a tu consumidor por lo que publica en Facebook y Twitter. Análisis de caso de ADT Security Services en Chile [Market research in social networks: know your consumer so publish on Facebook and Twitter. Case analysis of ADT Security Services in Chile] Vivat Academia. Revista de Comunicación, $\mathrm{n}^{\mathrm{o}}$ 144, 19-35. doi: http://doi.org/10.15178/va.2018.144.19-35. Recuperado

http://www.vivatacademia.net/index.php/vivat/article/view/1073

\section{INTRODUCCIÓN}

En la era de internet y de la hiperconectividad resulta lógico que, para conocer y contactar al consumidor, haya que recurrir a las redes sociales. Las estadísticas sobre penetración y uso los social media que se publican año tras año así lo ponen de relevancia: según el Estudio Anual de Redes Sociales 2016 editado por la consultora IAB Spain, un $94 \%$ de las personas en el mundo con acceso a internet cuenta con al menos un perfil en Facebook. Como segunda aplicación más empleada se sitúa Whatsapp, de uso recurrente para casi 9 de cada 10 internautas; Youtube cierra el podio al atraer a un $66 \%$ de usuarios de internet, manteniendo su liderazgo como portal de visionado de material audiovisual. En cuarto lugar y con un porcentaje de penetración sobre el $50 \%$ se posiciona Twitter.

En este contexto conviene destacar el papel preponderante de Facebook en las rutinas digitales de los internautas. Es más, el informe de tendencias digitales de eMarketer 2016, pronostica que para 2019 la penetración de esta red social se mantendrá estable, con una presencia aproximada del $80 \%$ entre público de 12 y 24 años, sin todavía evidencia empírica de que los jóvenes vayan a estar menos apegados a Facebook.

Tal es la relevancia que han ganado las redes sociales entre el público joven que la última versión del informe emitido en 2016 por el Instituto Reuters para el Estudio del Periodismo (RISJ) que explora y analiza el panorama informativo, constató que un $12 \%$ del total de la muestra (50.000 personas de 26 países) toma como principal fuente de información las redes sociales, uso que asciende al $28 \%$ en los jóvenes de 18 a 24 años. Igualmente, más de la mitad de las personas encuestadas (51\%) confirmó usar las redes sociales como fuente de noticias cada semana.

Del conjunto de redes sociales, Facebook se convierte en referente "para encontrar, leer, ver y compartir noticias" (RISJ, 2016). Tal como destaca el informe, conviene señalar que la televisión sigue siendo la principal fuente de consumo de noticias para los grupos de más edad, pero su uso continúa decreciendo especialmente entre el perfil más joven.

Ante estos datos de uso que ponen de relevancia el papel protagonista que han ido adquiriendo las redes sociales en la vida de los millenials, se hace necesario que las investigaciones sobre el comportamiento del consumidor se orienten hacia las plataformas en las que efectivamente están interactuando de forma frecuente. 


\subsection{Las redes sociales como fuente de investigación social}

Según lo expuesto, resulta evidente que se debe contactar con el usuario en esta esfera digital. En el contexto publicitario, la red debe concebirse como lugar de encuentro entre marcas y usuarios en donde la comunicación, para que funcione, se debe diseñar horizontalmente, de igual a igual, con el objetivo de crear un vínculo directo, estable y de confianza con la audiencia y que genere no solo el deseo de interactuar con la marca sino también el deseo de compartir contenido (Sánchez Torres \& Restrepo, 2015). Se trata de crear engagement "que es algo más complejo que generar un me gusta o retweets [...] Los mercadólogos deben tener claras las percepciones de sus consumidores, sus deseos, necesidades y preferencias, conectarse con ellos en sus propios términos" (2015, p.45).

Para lograr esa conexión con el consumidor, las marcas deben moverse al "ritmo vertiginoso" con el que el público actúa por la red "donde las decisiones se toman en segundos, porque cada vez los usuarios están más conectados al mundo, cuentan con un mayor acceso a la información y no solo son consumidores de contenido sino productores del mismo. Se han convertido en prosumidores hyperconectados e hyperinformados" (Sánchez Torres \& Restrepo, 2015, p.34). De ahí que una de las claves en la red sea actuar en tiempo real implementando estrategias de real time marketing. En 2015, según el estudio Real Time Marketing Report de Monetate y Econsultancy, un $70 \%$ de las empresas reconoció que sus acciones de marketing no eran lo suficientemente rápidas, una cualidad que en estos momentos puede ser vital para ganar presencia digital, fidelizar y captar nuevos consumidores.

Más allá de la improvisación, las marcas deben estudiar el comportamiento estudiar el comportamiento en las redes sociales de los consumidores y tener la capacidad de ajustar el trabajo de comunicación, atención y comercialización a las exigencias del momento, con un profundo conocimiento del mundo real y el virtual (Asociación Colombiana de Mercadeo, 2015, citado en Sánchez Torres \& Restrepo, 2015).

Consecuentemente, la investigación social se postula como el principal recurso para combatir cualquier intento de improvisación, error más frecuente en el entorno digital dado el volumen y la rapidez con la que se intercambia información.

La cantidad de tendencias que se generan cada día en redes sociales en las que las marcas podrían participar son innumerables; no obstante, se debe evaluar de una manera profesional cuáles son las que importan a su audiencia y nicho en específico (2015, p. 43).

Las redes sociales se erigen como el espacio idóneo para observar, para investigar el mercado, para conocer las inquietudes de los usuarios, especialmente de aquellos situados entre los 18 y 30 años. "Conocer la personalidad de los demás es una habilidad esencial para tener relaciones sociales exitosas" (Martínez Valerio, 2016, p.149) y en esta era de la comunicación digital, las redes sociales se conciben como una plataforma en la que los jóvenes no solo se comunican entre sí, sino que 
muestran su personalidad de una forma más o menos explícita.

Desde que Facebook comenzó su expansión mundial sobre el 2006, resulta destacable el interés que esta red social ha generado entre la comunidad científica sobre todo en relación con la exposición de la personalidad (Stutzman, 2006; Grasmuck et al., 2008; Mayer y Puller, 2008; Pempek et al., 2009; Back et al., 2010; Goldbeck, 2011; Junco, 2011), con un porcentaje relevante de investigaciones centradas en el contexto académico y/o universitario.

Aunque existe cierta discrepancia sobre la veracidad de la identidad mostrada en los entornos online (Cáceres et al., 2009; Chen \& Marcus, 2012, concluyen que se produce una cierta transformación de la personalidad real en la red), sí que la mayoría de aportaciones coinciden en que los principales rasgos identificativos de una persona reflejados en Facebook se corresponden con los de su vida offline (Back et al., 2010; Gosling et al., 2011). En esta línea, los resultados fruto de una las investigaciones de mayor envergadura realizadas sobre Facebook (Kosinski, 2013) también señalan una relación directa entre la personalidad, el perfil de Facebook y las webs visitadas. Para este estudio, los autores diseñaron una aplicación, My Personality, para obtener información sobre la personalidad de casi 7.5 millones de usuarios basada en el análisis de sus "Me gusta" en Facebook (Parra et al., 2014).

Lo cierto es que recientes investigaciones siguen apuntando en esta dirección de la autenticidad y honestidad de las manifestaciones publicadas en redes sociales al demostrar que "es posible conocer mejor a una persona incluso mejor que su propia familia a través de los datos que proporcionan en redes sociales" (Youyou et al., 2015, citado en Martínez Valerio, 2016, p.152).

En el contexto español son varios los estudios científicos que recurren a la observación de los "Me gusta" de Facebook para determinar pautas de comportamiento entre los usuarios. Una de ellas, realizada con la Dirección General de Tráfico, permitió mostrar la utilidad de la red social para la caracterización sociocultural de perfiles de conductores como paso previo al diseño de campañas publicitarias de sensibilización y prevención al volante (Parra et al., 2014); otra investigación en esta línea, de reciente publicación (Martínez Valerio, 2016), reflexiona sobre la posibilidad de conocer la personalidad de un perfil de usuario universitario analizando las páginas web a las que han dado "Me gusta".

Este trabajo en concreto también busca conocer el posicionamiento de los usuarios hacia un determinado concepto de forma no inductiva, pero en este caso recurriendo al análisis de los comentarios que hacen espontáneamente en las redes sociales (Facebook y Twitter). Se parte de la idea de que el análisis de contenido en redes sociales se postula como una vía más directa y fidedigna para definir pautas de comportamiento que por otras técnicas (encuesta, entrevista...) resultan más difícil de detectar.

Hasta la fecha la investigación en redes sociales ha privilegiado el análisis estadístico de audiencias digitales y trending topics a partir de herramientas prediseñadas como Google Analytics o Sharedcount. La mayoría de estas aplicaciones analíticas facilitan el tratamiento estadístico de la información sin llegar a profundizar en los contextos de producción y significado social de los datos (Parra et al., 2014, p.197). 
Las principales redes sociales en las que se realizan investigaciones de mercado son Twitter y Facebook ya que se posicionan como las dos mayores redes de información en las que se comparte más contenido y en las que más rápidamente los internautas publican sus comentarios.

Una parte importante de la información que Facebook conoce sobre sus usuarios la proporcionan ellos mismos voluntariamente, complementada con otro volumen de datos que se puede obtener a través de su actividad en la red social, pudiendo conformar un perfil bastante completo de cada usuario. Cuestión similar sucede en Twitter, donde la mayoría de personas hacen tweets públicos, lo que posibilita la creación de bases de datos sobre potenciales clientes que se pueden tratar científicamente mediante la propia observación (rastreando personas, empresas, grupos, listas o hashtags).

Esta investigación pretende ir un paso más allá del de determinar la personalidad de los usuarios a través de sus comentarios en redes sociales. Se busca que esa información digital analizada sirva de guía para el diseño de estrategias creativas de las marcas, cubriendo un nicho de investigación latente que vincule el estudio de audiencias en redes sociales con la efectividad publicitaria. La mayoría de aportaciones sobre investigación en redes sociales centran sus esfuerzos en estudiar contenidos generados por un determinado público. Montero (2013) diferenció cinco líneas de estudio: redes sociales y dinámicas de aprendizaje; redes sociales y medios de comunicación; gestión de contenidos en redes sociales; redes sociales y audiencia y mercados y redes sociales. La autora señaló la necesidad de integrar en este tipo investigaciones en redes sociales una utilidad publicitaria para fomentar los procesos de interacción entre audiencias digitales y anunciantes. "Se estaría hablando de un sexto enfoque relacionado con las redes sociales, audiencias y marcas" (Montero, 2013, p.50).

Es en esta línea que propone Montero donde se integra este trabajo de investigación en el que se toman como fuente creativa los insights publicados (durante tres meses) por el potencial público objetivo de una marca (chilenos que comenten sobre temas de seguridad en redes sociales), en este caso ADT Security Services.

\subsection{La investigación como base para la estrategia creativa}

Desde estas líneas se defiende la necesidad de una creatividad aplicada, fruto de un proceso previo de investigación y reflexión.

La investigación científica en creatividad publicitaria puede parecer fuera de lugar en una actividad tan poco sometida a lógica de razón y a las dudas existenciales de la ciencia. Sin embargo, la investigación científica en Creatividad Publicitaria es necesaria para elaborar mensajes capaces de llamar la atención del receptor y alcanzar los objetivos publicitarios (Arroyo, 2015).

Intuitivamente se puede pensar que la creatividad en publicidad y en otras ramas del saber supone un fenómeno caprichoso que se presenta cuando menos se la espera 
y de la mano de individuos con mentes privilegiadas. No obstante, con la experiencia se hace más patente que una idea creativa es sinónimo de utilidad, de resolución de problemas, un estado al que se llega tras una reflexión profunda basada en un proceso de investigación previo.

Se debe buscar en la realidad entendiendo el proceso creativo como un método que permita al publicista llegar a una verdad útil que represente y enganche a su interlocutor. Para autores como Bunge, Bochenski o Gil Múñoz (citados en Arroyo, 2015) la creatividad publicitaria es una trayectoria mental "entendiéndola como una serie de operaciones mentales efectuadas en el espacio de representación del problema de un anunciante" $(2015$, p.1).

Tal como propone Arroyo siguiendo a Francisco García (1987), la creatividad se concibe como un método científico -útil, instrumental, regular, objetivo, razonable (Sierra Bravo, 2001)- que debe responder a las siguientes fases de investigación: definición del problema de comunicación; documentación, en este caso a través de redes sociales; formulación de objetivos e hipótesis sobre la originalidad y efectividad de la propia idea; la verificación de dichas hipótesis, que en creatividad publicitaria tendrán especial importancia aquellas técnicas propias de las ciencias sociales $\mathrm{y}$, finalmente evaluación y presentación de los resultados,

etapa creativa de la difusión del invento y tiene un claro sentido socializador [...] No basta que el invento sea aceptado por los expertos, es necesario que trascienda al público. Y cuando se trata de la Creatividad Publicitaria, con muchísima más razón [...] Es importante que los resultados de la investigación encuentren su aplicación, ya sea teórica o práctica (Arroyo, 2015, p.12).

Asimismo, la creatividad aplicada no la alcanza un individuo de forma aislada, al estilo de "llanero solitario", se trata de un proceso que se debe entender en equipo, trabajando conjuntamente sobre todo en el campo publicitario en el que la multiplicidad de puntos de vista no puede más que enriquecer la utilidad de esa idea inicialmente propuesta.

\section{OBJETIVOS}

El estudio de caso se presenta como parte de una investigación realizada para la empresa ADT Security Services Chile, filial de la multinacional Tyco. Los objetivos generales de esta investigación fueron la búsqueda e identificación de hallazgos, clústeres de información, insight y actitudes generales que movilicen la compra y consumo de seguridad en el mercado señalado, y profundizar en éstas para una posterior campaña estratégica y creativa a partir de una metodología que incorpora el uso de las redes sociales Facebook y Twitter.

El análisis proviene de una estudio cuantitativo y cualitativo que responde a la necesidad de entender y conocer a cabalidad a los chilenos respecto de los temas de seguridad para así poder definirlos y caracterizarlos desde las conversaciones en torno al tema de la seguridad en Chile, y con esto, poder orientar el diseño de la estrategia creativa, de marketing y comunicacional de dicha empresa. 


\section{METODOLOGÍA}

La metodología se bautizó con el nombre de The Orange Method, de carácter cualitativo, basada en el paradigma fenomenológico que busca entender el significado de las acciones humanas y de la práctica social. Se trata de una variante de la Netnografía, nueva disciplina que se está desarrollando para entender la realidad social que se produce en el mundo digital (Del Fresno, 2011). Es entonces una etnografía adaptada al estudio de las comunidades en línea (Hine, 2000) método más rápido, más sencillo y menos costoso que la etnografía tradicional, y más naturalista y discreto que los grupos de discusión o entrevistas. Pero en este caso se obtiene información pública no inductiva sobre el simbolismo, los significados y los patrones de consumo de grupos de consumidores en línea sin preguntarles, sino que desde el análisis de los comentarios que estos hacen espontáneamente en la web.

Se utilizó específicamente en este caso para el análisis de declaraciones espontáneas realizadas a través de las redes sociales Facebook y Twitter por hombres y mujeres chilenos entre los meses de octubre a diciembre del año 2015, acerca de temas relacionados con la seguridad.

Los datos se obtuvieron utilizando un robot de búsqueda ontológica a través del uso de keywords relacionadas con la palabra y el concepto de seguridad, facilitado por la empresa líder y pionera en Chile en la creación de una plataforma comprehensiva de captura, procesamiento y generación de métricas para el análisis de la información de redes sociales Brandmetric.

El diseño metodológico consta de cinco fases consecutivas:

1. Búsqueda: Recolección de comentarios espontáneos de consumidores en la web (redes sociales, web, blogs, etc) a través de un software de búsqueda.

2. Análisis: Determinar los grandes clústeres de información dentro de las conversaciones analizadas.

3. Segmentar: Dividir los distintos grupos y/o motivaciones que movilizan los comentarios relacionados con la marca, producto, servicio, etc.

4. Empatizar: Entender las distintas motivaciones que movilizan a las personas a tomar decisiones y opinar o reaccionar de una u otra forma. Comprender las emociones contenidas detrás de los comentarios.

5. Identificación: Reconocimiento de insights, oportunidades de innovación, emociones y sensaciones que permitan trabajar vínculos potentes para desarrollar la creatividad y estrategia con mayor precisión.

\section{RESULTADOS}

De acuerdo al diseño metodológico planteado se siguieron las cinco fases de trabajo de análisis: 


\subsection{Búsqueda}

La muestra resultante fue de 321.169 comentarios recogidos, de los cuales el 59,9\% (123.255 comentarios) correspondieron a personas que en su perfil o cuenta de la red social utilizada se identificaron como hombres, y el 40,1\% (82.421 comentarios) correspondieron a personas que en su perfil o cuenta de la red social utilizada se identificaron como mujeres.

En cuanto a la distribución por canal de comunicación, el 59,3\% del total de los comentarios emitidos fueron a través de la red social Facebook, mientras que el 40,7\% lo hizo desde la red social Twitter.

\subsection{Análisis}

Una vez recogidos los datos, estos fueron divididos en los grandes grupos conceptuales a los que pertenecían dividiéndose así en: alarma, asalto, robo, delincuencia, seguridad, portonazo y hurto.

\subsection{Segmentar}

Luego de la división por grupos se segmentaron los comentarios de acuerdo a las diferentes motivaciones que movilizaron a las personas a dejar su opinión en las redes sociales: sensación de inseguridad; sentimiento de impotencia; el robo como algo rutinario; desconfianza en autoridades, empresas e instituciones; preocupación; solución en sus manos y la necesidad de organizarse y agruparse.

\subsection{Empatizar}

Análisis comprehensivo de los diferentes segmentos de comentarios identificados:

1. Sensación de inseguridad: Los comentarios de este segmento aludieron a un sentimiento de inseguridad en cualquier lugar, las personas no se sienten seguras ni en lugares públicos, como tampoco privados. Comentarios como: "En el mall Alto Las Condes no hay seguridad para entrar en cualquier horario, cualquiera tiene libre acceso"; "falta seguridad en mi barrio @Barrio_Yungay", reafirmaron este sentimiento. 


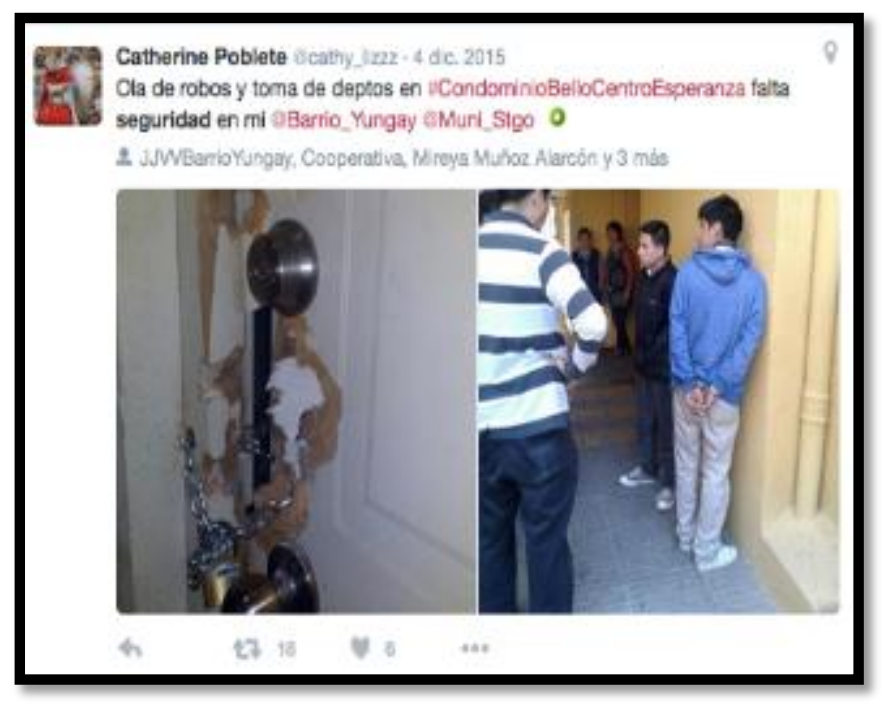

Foto 1: Captura de un tweet que evidencia esa sensación de inseguridad.

Fuente: Twitter

2. Sentimiento de impotencia: Las personas se observan con sentimientos de impotencia ante la inseguridad, en sus comentarios describen un problema que no tiene solución y que no son escuchados tanto por el gobierno y autoridades, como por las empresas sus requerimientos. "@Carabineros no atiende reclamo de vecinos", "a nadie le importa la seguridad", "nadie se hace cargo", "hoy es legítimo robar en Chile" (apoyado por material visual de causas legales). 


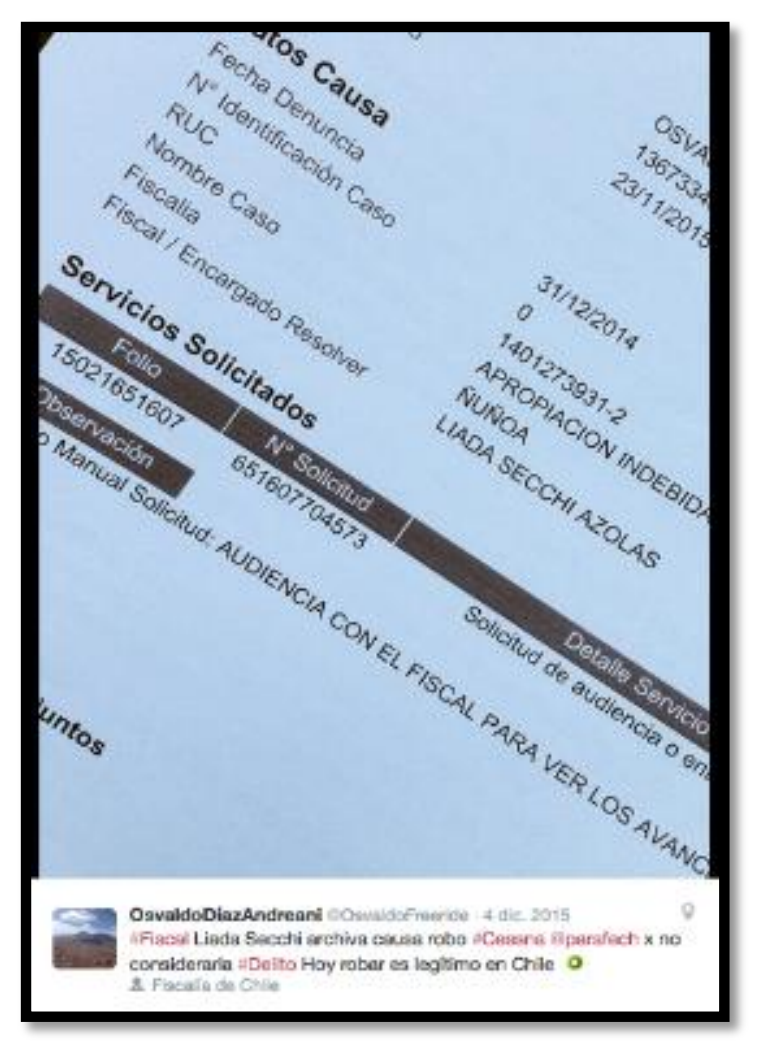

Foto 2: Captura de un tweet que ejemplifica el sentimiento de impotencia.

Fuente: Twitter

3. El robo como algo rutinario: En sus comentarios se logra observar una costumbre al robo, algo que pasa a ser de la rutina diaria, un hecho que no sorprende y que se empieza a ver como algo cotidiano. "a pesar del bajón por el robo, me tengo fe con mi alegato de hoy", "esperando que me atiendan mientras pienso en el robo que me hicieron ayer, que mala pata", "después de que me robaron pasé donde Francisca".

4. Desconfianza en autoridades, empresas e instituciones: Los comentarios demostraban una falta de confianza y fiabilidad en la competencia y capacidad de resolver problemas de autoridades, empresas e instituciones. Memes o comentarios como: "da lo mismo la alarma si igual nadie se hace cargo", "y en las próximas elecciones nuevamente van a prometer seguridad", "Delincuentes se les acabó la fiesta dijo un ex presidente y nada hizo", "el problema de la seguridad trasciende hace más de 40 años". 


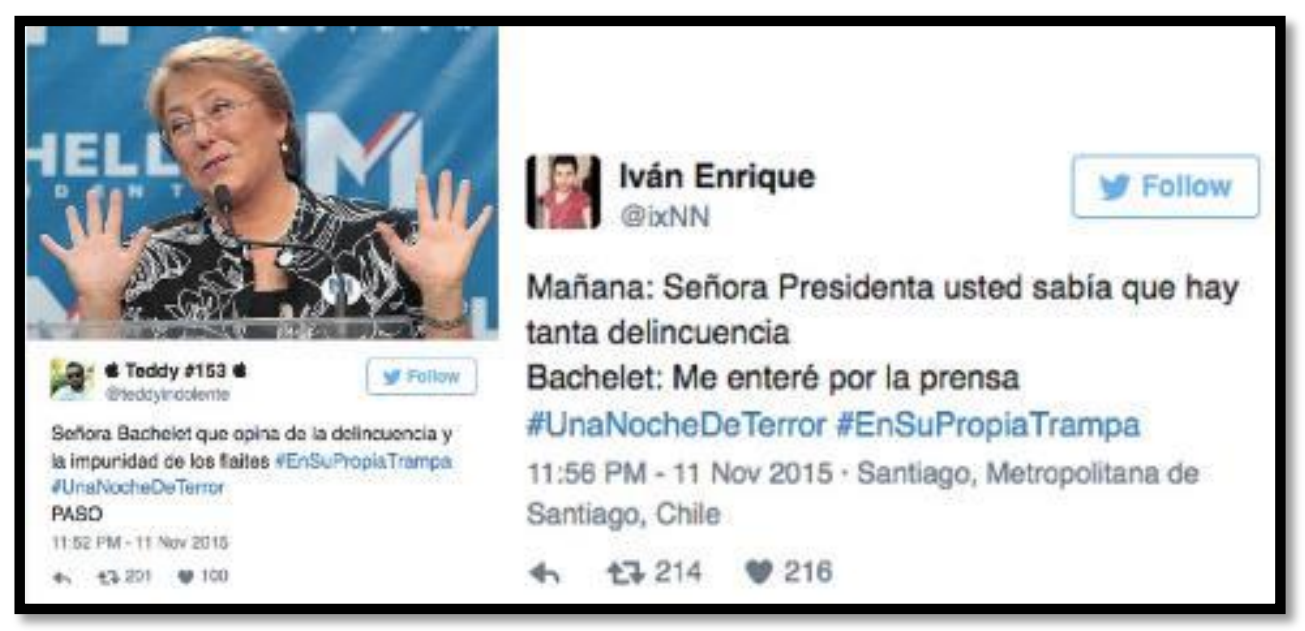

Foto 3: Capturas de Twitter en las que los ciudadanos muestran su falta de confianza en el gobierno chileno. Fuente: Twitter

5. Preocupación: El robo se posiciona como una de las mayores preocupaciones, se convirtió en tendencia en Twitter y se observa que empieza a afectar la calidad de vida de los chilenos.

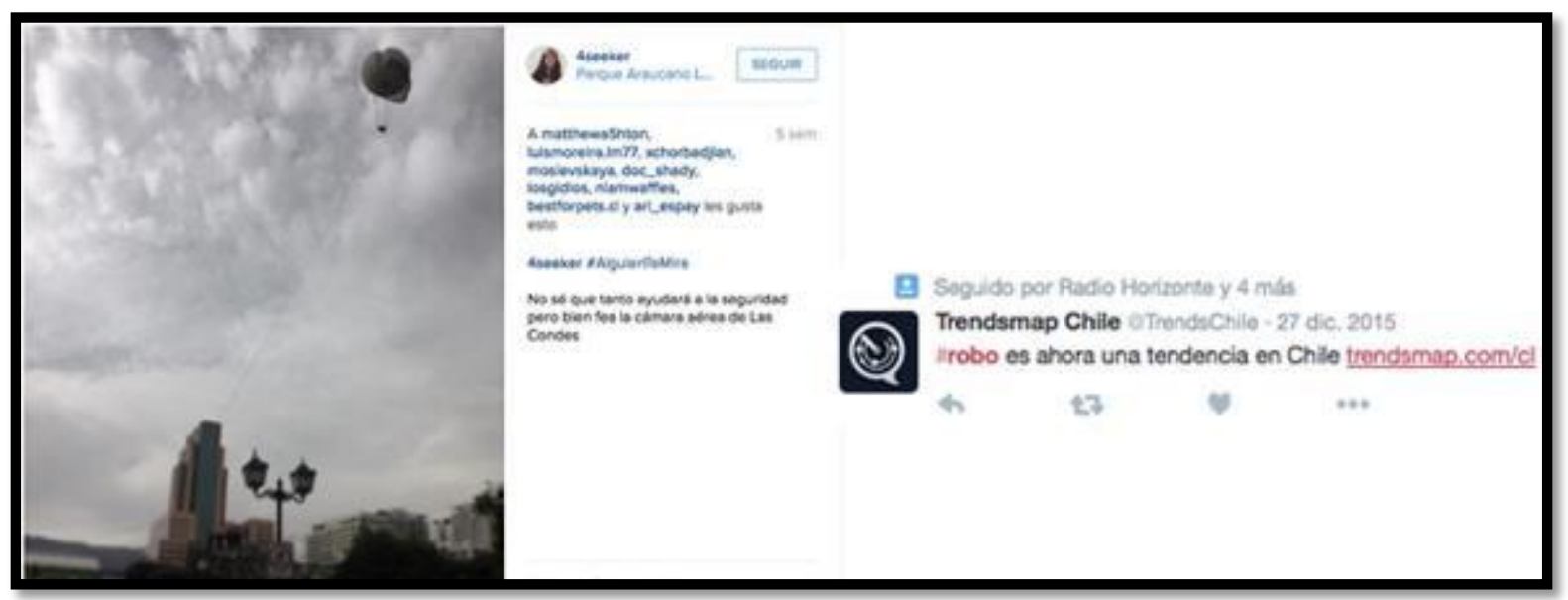

Foto 4: Ejemplos de comentarios en Facebook y Twitter en los que el robo es una preocupación de peso entre la ciudadanía. Fuente: Facebook y Twitter

6. Solución en sus manos: Creen que la solución puede provenir desde ellos mismos y empiezan a demostrar por medio de diferentes acciones retratadas en imágenes y comentarios, que ellos mismos son capaces de hacerle frente a los robos y tomar la justicia en sus manos. 


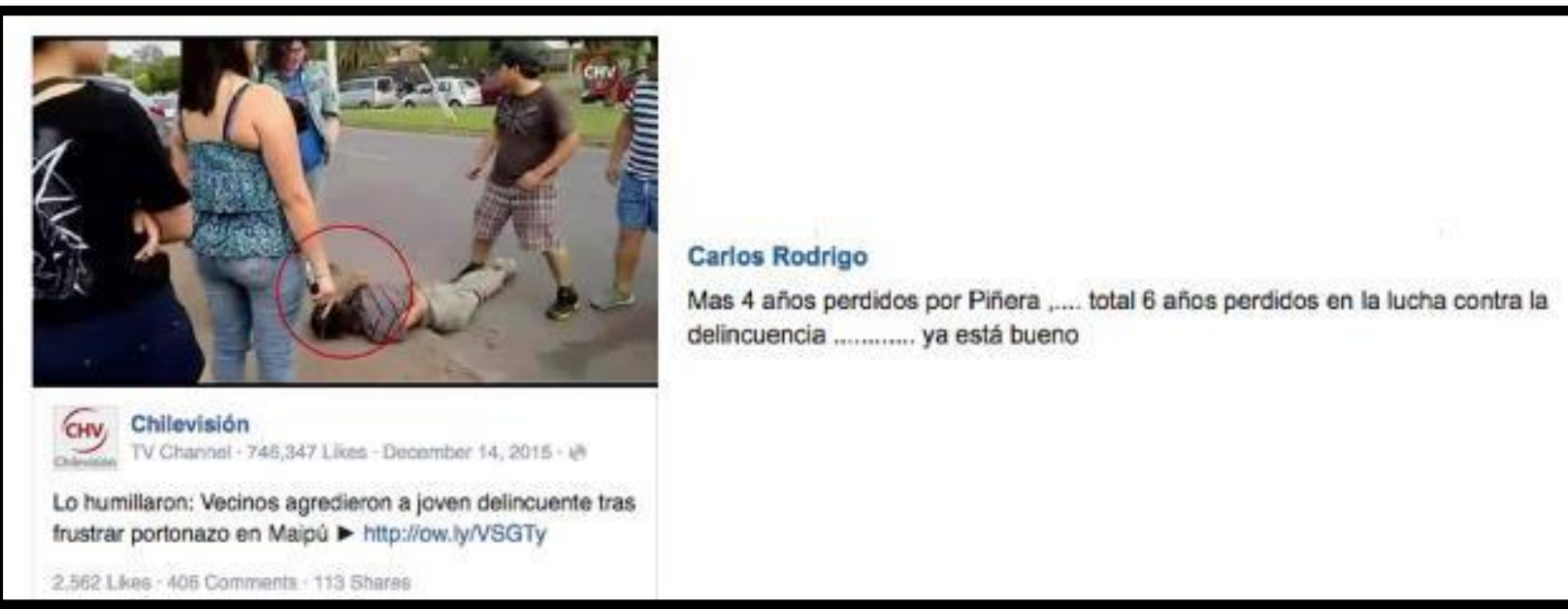

Foto 5: Entradas de Facebook que exhiben la proactividad de las personas anónimas en materia de seguridad. Fuente: Facebook

7. Necesidad de organizarse y agruparse: Ante la impotencia y escasas soluciones, se empiezan a agrupar y reunir para hacer frente al problema. Para eso se valen de grupos de WhatsApp organizados por vecinos, apps de seguridad como SOSAFE y hacen exigencias a las autoridades organizados en grupos de vecinos.

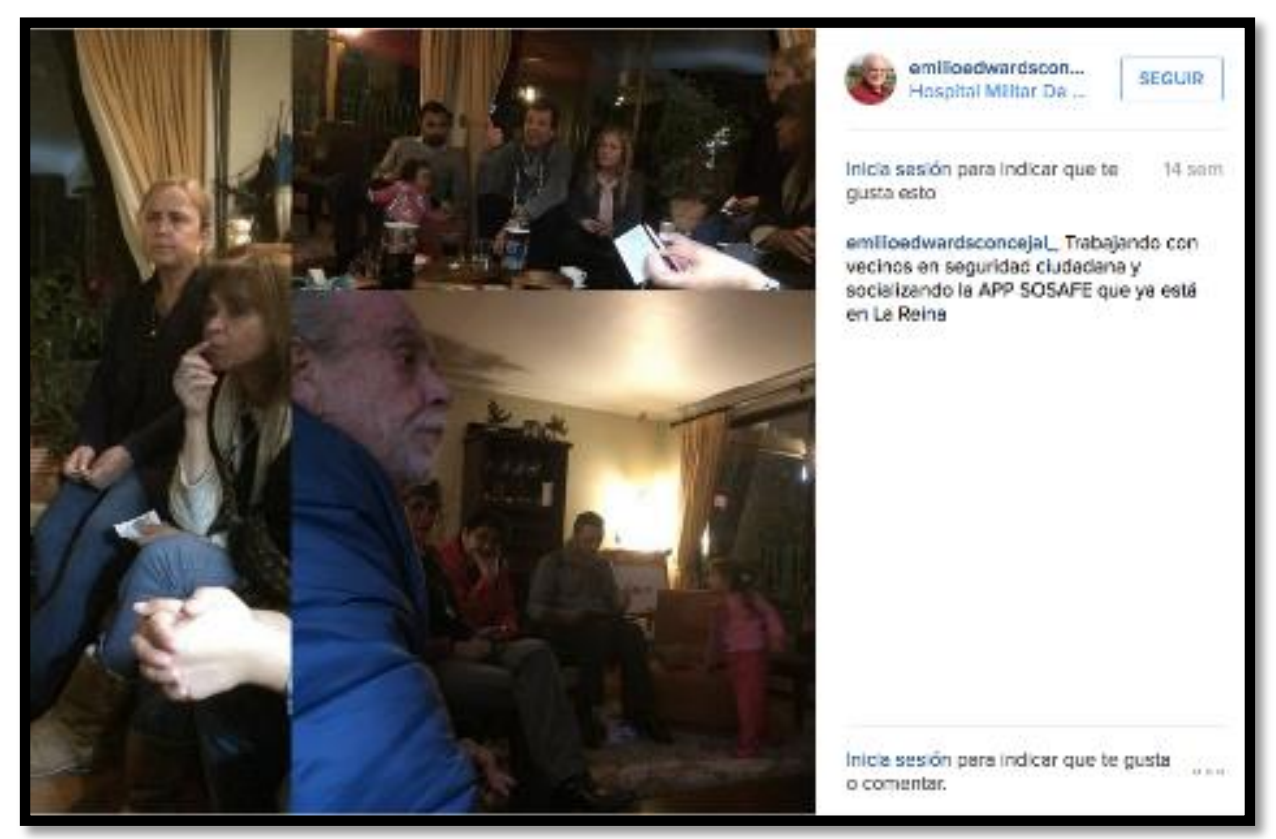

Foto 6: Entrada en Facebook con fotografías que ejemplifica como los vecinos se organizan para combatir la inseguridad. Fuente: Facebook 


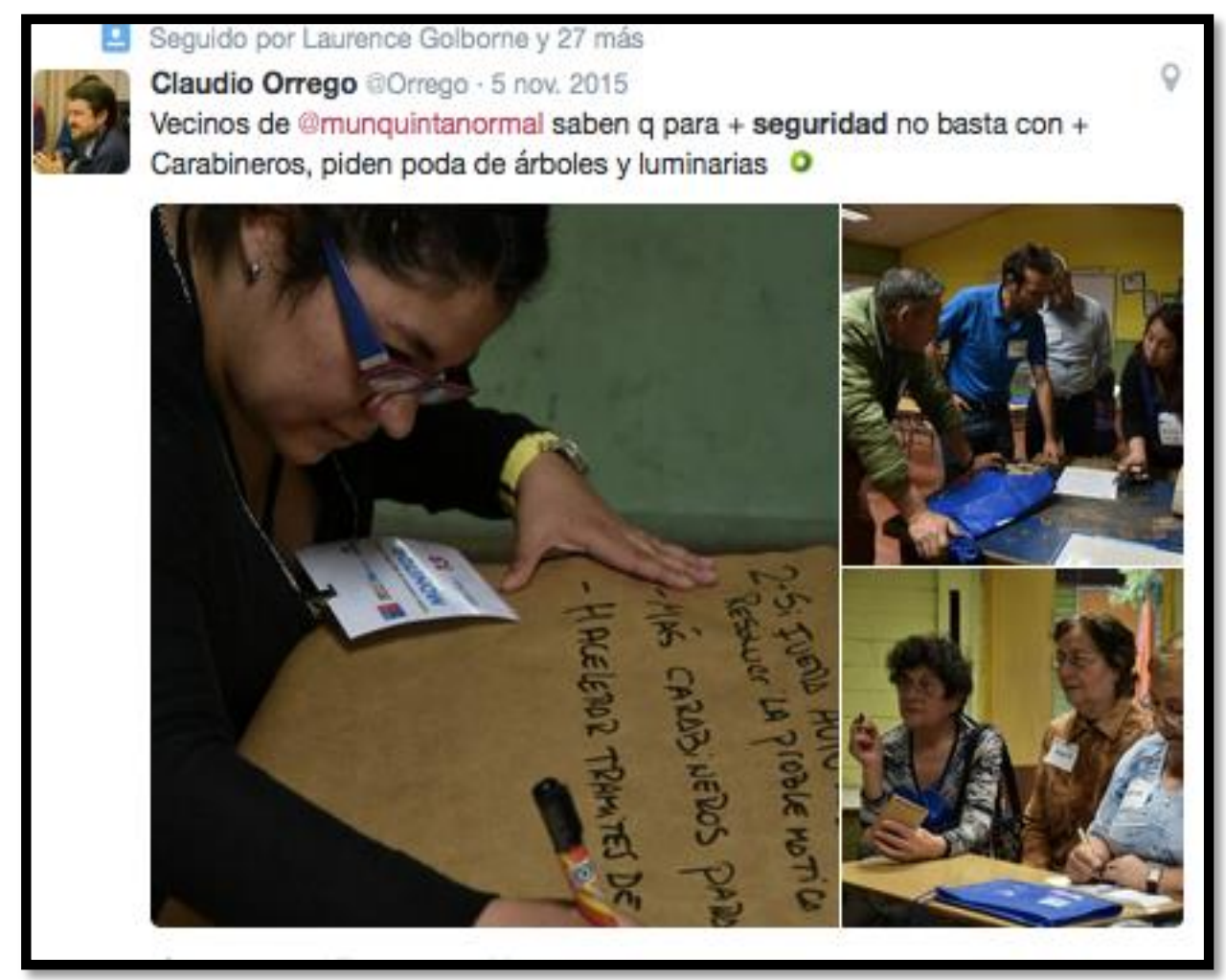

Foto 7: Entrada en Twitter con fotografías donde se evidencia el compromiso vecinal por conseguir barrios más seguros. Fuente: Twitter

\subsection{Identificación}

Se logró reconocer insights, oportunidades de innovación, emociones y sensaciones que permitieron trabajar vínculos potentes para desarrollar la creatividad y estrategia con mayor precisión.

Comentarios como: "la App le avisa a mis conocidos y vecinos", "tengo una alarma y me avisa al celular", "la casa ya tenía alarma" llevaron a entender el uso de alarmas, por qué no contratan, cómo y para qué las usan.

Respecto del robo los comentarios llevaron a entender cómo se están reuniendo y cuáles son sus procesos: "una vecina organizó un Whatsapp", "hay que estar atentos", "si pasa algo llamamos al fono emergencia de la municipalidad", "mi vecino nos avisó y pudimos pararlo".

Respecto de la contratación de empresas de seguridad se logró descubrir sentimientos, miedos y opiniones que influyen sobre cómo se comportan como consumidores de estas: "para que pagar mensual si no sirven para nada", "si suena la alarma te llaman por teléfono, y si te tienen encañonado?", "cuando llegan los de seguridad ni si quiera pueden entrar a la casa, no sirven".

\section{DISCUSIÓN}

Todo el proceso descrito llevó a la investigación a descubrir un cambio cultural en el país. Desde hace muchos años, desde la década de los 80, en los barrios socioeconómicos altos de Chile no es frecuente que se dé un contacto entre los 
vecinos, quienes viven en casas o departamentos no conocen, ni saludan, ni conversan con sus vecinos. No saben quién habita al lado, ni les interesa saberlo. Se vive prácticamente en "burbujas" donde es hasta poco probable ver un saludo en el ascensor de un edificio. Cada uno se preocupa de sí mismo y mientras menos contacto tenga con quienes lo rodean, mejor.

El problema de la inseguridad trajo consigo una mejora en la sociedad chilena, equiparó los barrios más populares con los barrios altos. Ante la imposibilidad de resolver el problema de la seguridad por parte de las autoridades, los vecinos se volvieron a conectar, se empezaron a conocer y agrupar ayudados por la tecnología.

Este descubrimiento fue lo que se comunicó a la agencia creativa y a la empresa de seguridad con lo que lograron idear una exitosa campaña publicitaria "vecino con vecino" que representa y recoge los sentimientos y actitudes más profundas de los chilenos frente a la seguridad (la pieza se puede visionar en https:// www.youtube.com/watch?v=I5f_I9pRg9s).

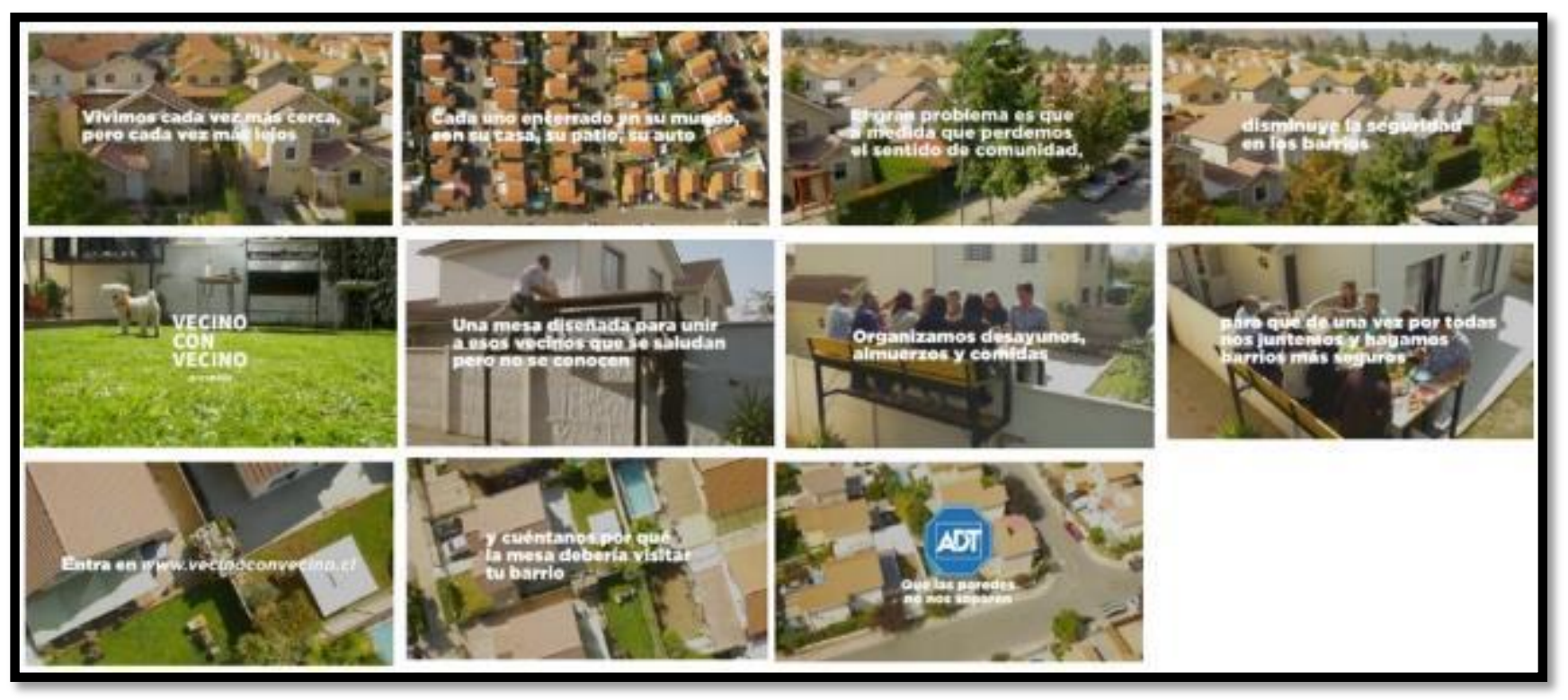

Foto 8: Frames extraídos de la campaña objeto de estudio. Fuente: https://www.youtube.com/watch?v=I5f_I9pRg9s

\section{CONCLUSIONES}

El diseño de investigación descrito perfila una metodología de trabajo que permite y se ofrece como complemento a la investigación científica de audiencias y sociedades para el posterior desarrollo creativo de comunicación o marketing para continuar mejorando la experiencia de los consumidores.

Por medio del análisis de redes sociales: las características sociales, conductas, opiniones, emociones, sentimientos y los patrones de uso frente a un concepto en particular, ha permitido profundizar en la caracterización sociocultural de los grupos objetivos identificando insights potentes para la realización de creatividad y estrategias. 
El modelo de análisis de datos The Orange permite aportar información adicional y relevante de los grupos de interés, en este caso de los chilenos frente al tema de la inseguridad, estableciendo un análisis integral y comprensivo de las preocupaciones y resistencias socioculturales en torno a un tema en particular para el diseño de campañas publicitarias y estrategias de marketing específicas.

\section{REFERENCIAS}

Arroyo, I. (2005). Metodología de la investigación científica en Creatividad publicitaria. Enlaces: Revista del CES Felipe II, 4, 1-12.

Back, M.; Stopfer, J.; Vazire, S.; Gaddis, S.; Schmukle, S.; Egloff, B. \& Gosling, S. (2010). Facebook Profiles Reflect Actual Personality, Not Self-Idealization. Psychological Science, 21(3), 372-374. doi: 10.1177/0956797609360756.

Cáceres, M.D.; Ruiz San Román, J.A. \& Brändler, G. (2009). Comunicación interpersonal y vida cotidiana. La presentación de la identidad de los jóvenes en Internet. Cuadernos de Información y Comunicación, 14, 213-231.

Chen, B. \& Marcus, J. (2012). Students' self-presentation on Facebook: An examination of personality and self-construal factors. Computers in Human Behavior, 28(6), 2091-2099. doi:10.1016/j.chb.2012.06.013.

Del Fresno, M. (2011). Netnografía. Barcelona: UOC.

Econsultancy \& Monetate. (2014). Real-Time Marketing Report. Nueva York: Centaur Media. Recuperado de https:/ / econsultancy.com/reports/real-time-marketing-report/

Elogia Media. (2016). Estudio Anual de Redes Sociales. Madrid: IAB Spain. Recuperado de

http://www.iabspain.net/wpcontent/uploads/downloads/2016/04/IAB_EstudioRedesSociales_2016_VCorta.pdf

Emarketer. (2015). Tendencias del Marketing Digital 2015. Santiago de Chile: IAB Chile. Recuperado de http://www.iab.cl/tendencias-marketing-digital-2015-emarketer/

Golbeck, J.; Robles, C. \& Turner, K. (2011). Predicting personality with social media. En CHI '11 Human Factors in Computing Systems (pp. 253-2262). NY, USA: ACM New York. doi: 10.1145/1979742.1979614.

Gosling, S.; Augustine, A.; Vazire, S.; Holtzman, N. \& Gaddis, S. (2011). Manifestations of Personality in Online Social Networks: Self-Reported Facebook-Related Behaviors and Observable Profile Information. Cyberpsychology, Behavior, and Social Networking, 14 (9), 483-488. doi:10.1089/cyber.2010.0087.

Grasmuck, S.; Martin, J. \& Zhao, S. (2008). Identity construction on Facebook: Digital empowerment in anchored relationships. Computers in Human Behavior, 24(5), 1816-1836. doi: 10.1016/j.chb.2008.02.012

Hine, C. (2004). Etnografía virtual. Barcelona: UOC.

Junco, R. (2011). The relationship between frequency of Facebook use, participation in Facebook activities, and student engagement. Computers and Education, 58(1), pp.162-171. doi: 10.1016/j.compedu.2011.08.004

Kosinski, M.; Stillwell, D.; \& Graepel, T. (2013). Private traits and attributes are predictable from digital records of human behavior. Proceedings of the National Academy of Sciences, 110(15), 5802-5805. doi: 10.1073/pnas.1218772110 
Feijoo Fernández, B.; Guerrero, J. J. La investigación de mercados en redes sociales: conoce a tu consumidor por lo que publica en Facebook y Twitter. Análisis de caso de ADT Security Services en Chile

Martínez Valerio, L. (2015). Conocer la personalidad de los universitarios a través de los "me gusta" de Facebook. Prisma Social: revista de ciencias sociales, 15, 147-179.

Mayer, Z. \& Puller, S. (2008). The old boy (and girl) network: Social network formation on university campuses. Journal of Public Economics, 92, 329-347. doi: 10.1016/j.jpubeco.2007.09.001

Montero, L. (2013). Reflexiones sobre la investigación en redes sociales: Facebook y Twitter. En Actas I Congreso Internacional de Comunicación y Sociedad Digital (pp. 41-52). Logroño: UNIR.

Parra, P.; Gordo, A.J. \& D’Antonio, S.A. (2014). La investigación social aplicada en redes sociales. Una innovación metodológica para el análisis de los «Me gusta» en Facebook". Revista Latina de Comunicación Social, 69, 195-212. doi: 10.4185/RLCS-2014-1008

Pempek, T.; Yermolayeva, Y. \& Calvert, S. (2009). College students' social networking experiences on Facebook. Journal of Applied Developmental Psychology, 30(3), $227-$ 238. doi: 10.1016/j.appdev.2008.12.010

Reuters Institute for the Study of Journalism. (2016). Reuters Institute Digital News Report 2016. Oxford: University of Oxford. Recuperado de http://reutersinstitute.politics.ox.ac.uk/sites/default/files/Digital-News-Report-2016.pdf

Sánchez Torres, W.C. \& Restrepo, J.C. (2015). Marketing en tiempo real y el fortalecimiento del mercadólogo. Escenarios: empresa y territorio, 4(4), 33-52.

Sierra Bravo, R. (2001). Técnicas de Investigación Social. Teorías y ejercicios. Madrid: Ediciones Paraninfo S.A.

Stutzman, F. (2006). An evaluation of identity-sharing behavior in social network communities. Journal of the International Digital Media and Arts Association, 3(1), 10-18.

\section{AUTORES}

\section{Beatriz Feijoo Fernández}

Doctora en Comunicación y licenciada en Publicidad y Relaciones Públicas y Comunicación Audiovisual por la Universidade de Vigo (España). Ejerce de docente e investigadora en la Facultad de Comunicación de la Universidad de los Andes (Chile). También ha estado vinculada profesionalmente al campo del periodismo y de la comunicación institucional al trabajar durante varios años en el Área de Comunicación de la Universidade de Vigo. Autora de diversas publicaciones y artículos científicos sobre comunicación y niños.

http://orcid.org/0000-0001-5287-3813

\section{Juan José Guerrero Cortés}

Licenciado en Publicidad por la Universidad del Pacífico (Chile), Máster en Comportamiento del Consumidor por la Universidad Adolfo Ibañez (Chile) y Doctorando en Comunicación en la Universidad de Los Andes (Chile). Ejerce de docente e investigador en la Facultad de Comunicación de la Universidad de los Andes. Profesionalmente se desempeñó más de 9 años como director Creativo en agencias de Publicidad y como Director Comercial y Gerente General en diferentes Consultoras de Marcas. https://orcid.org/0000-0002-7286-3660 